\title{
Plasmacytic Post-transplant Lymphoproliferative Disorder - Case Report
}

\author{
Jiri Hanousek, ${ }^{1}$ Jakub Radocha, ${ }^{1}$ Ondrej Soucek, ${ }^{2}$ Lenka Pliskova, ${ }^{3}$ Katerina Kamaradova, ${ }^{4}$ \\ Alzbeta Zavrelova' and Pavel Zak' ${ }^{1}$ \\ 1. 4th Department of Internal Medicine - Haematology, University Hospital Hradec Kralove and Charles University in Prague, Faculty of Medicine in Hradec \\ Kralove, Hradec Kralove, Czech Republic; 2. Institute of Clinical Immunology, University Hospital Hradec Kralove and Charles University in Prague, Faculty of \\ Medicine in Hradec Kralove, Hradec Kralove, Czech Republic; 3. Institute of Clinical Biochemistry, University Hospital Hradec Kralove and Charles University \\ in Prague, Faculty of Medicine in Hradec Kralove, Hradec Kralove, Czech Republic; 4. The Fingerland's Department of Pathology, University Hospital Hradec \\ Kralove and Charles University in Prague, Faculty of Medicine in Hradec Kralove, Hradec Kralove, Czech Republic
}

DOI: https://doi.org/10.17925/EOH.2017.13.01.80

W e present a case of a 43-year-old Caucasian female with acute myeloid leukaemia (AML), who developed Epstein-Barr virus (EBV) positive post-transplant lymphoproliferative disorder (PTLD) of duodenum, with plasma cell differentiation after second haematopoietic stem cell transplantation. The patient was given rituximab and CHOP chemotherapy (cyclophosphamide, doxorubicin, vincristine and prednisone); however, these were only temporarily effective, and the patient passed away 36 days after PTLD was diagnosed. PTLD with plasma cell differentiation is a rare type of PTLD, and there are no strict treatment guidelines.

\section{Keywords}

Plasmacytoma, post-transplant lymphoproliferative disorder, PTLD, haematopoietic stem cell transplantation, HSCT

Disclosure: Jiri Hanousek, Jakub Radocha, Ondrej Soucek, Lenka Pliskova, Katerina Kamaradova, Alzbeta Zavrelova and Pavel Zak have nothing to disclose in relation to this article. No funding was received in the publication of this article.

Ethics: All procedures were followed in accordance with the responsible committee on human experimentation and with the Helsinki Declaration of 1975 and subsequent revisions, and informed consent was received from the patient involved in this case study.

Authorship: All named authors meet the International Committee of Medical Journal Editors (ICMJE) criteria for authorship of this manuscript, take responsibility for the integrity of the work as a whole, and have given final approval to the version to be published.

open Access: This article is published under the Creative Commons Attribution Noncommercial License, which permits any non-commercial use, distribution, adaptation and reproduction provided the original author(s) and source are given appropriate credit.

Received: 1 February 2017

Accepted: 2 May 2017

Citation: European Oncology \& Haematology, 2017;13(1):80-2

Corresponding Author: Jiri Hanousek, IV interni hematologicka klinika, Sokolska 581, Hradec Kralove 500 05, Czech Republic. E: jiri.hanousek@fnhk.cz
Post-transplant lymphoproliferative disorder (PTLD) is a severe complication of haematopoietic stem cell transplantation (HSCT). Most of the PTLDS originate from B-cells. Overall, $60-80 \%$ of PTLDs are associated with Epstein-Barr virus (EBV); this can vary depending on PTLD type and time of onset after HSCT.1,2 The majority of the general population is infected by EBV during their life, but an immunocompetent host mounts a swift immune response that controls proliferation of EBV infected B-cells. EBV then resides only in resting memory B-cells in latent form. ${ }^{3}$ The balance between latently infected B-cells and immune surveillance is disrupted after a patient receives HSCT. An increase in the number of infected B-cells (almost always of donor origin) may develop into PTLD. ${ }^{4-6}$

PTLDS are divided into three stages of evolution: early lesions, polymorphic PTLDS and monomorphic PTLDS. Monomorphic PTLDs are mostly derived from B-cells, but can also be of T-cell origin, and resemble aggressive non-Hodgkin lymphomas. ${ }^{5}$ PTLD resembling diffuse large

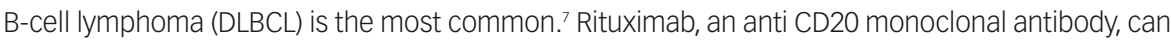
be used effectively to treat them. ${ }^{8.9}$ Other types of PTLD are rare and only $4 \%$ of them show plasma cell differentiation. 7,10 We would therefore like to present our patient with PTLD of duodenum with plasma cell differentiation.

\section{Case report}

A 43-year-old Caucasian female was diagnosed with MLL/PTD- and FLT3/ITD-positive acute myeloid leukaemia (AML) in June 2013. She was given " $3+7$ " induction chemotherapy regimen (cytarabine $100 \mathrm{mg} / \mathrm{m}^{2}$ for 7 days and daunorubicin $90 \mathrm{mg} / \mathrm{m}^{2}$ for 3 days) followed by consolidation chemotherapy high-dose cytarabine (HIDAC) in July 2013. She achieved complete remission (CR) with negative minimal residual disease (polymerase chain reaction [PCR] for MLL/PTD gene). She underwent allogeneic HSCT with 10/10 sibling donor (sister) in September 2013, using a conditioning regimen of busulfan $3.2 \mathrm{mg} / \mathrm{kg}$ for 4 days, fludarabine $30 \mathrm{mg} / \mathrm{m}^{2}$ for 5 days and rabbit derived anti-thymocyte globulin ( $\mathrm{r}$-ATG [Thymoglobulin; Sanofi, Paris, France]) $3 \mathrm{mg} / \mathrm{kg}$ for 1 day, with tacrolimus and mycophenolate mofetil as immunosuppression. She remained in CR for 14 months but relapsed in November 2014. She was then given Flag-Ida chemotherapy (fludarabine $30 \mathrm{mg} / \mathrm{m}^{2}$ for 5 days, cytarabine $2 \mathrm{~g} / \mathrm{m}^{2}$ for 5 days and idarubicin $12 \mathrm{mg} / \mathrm{m}^{2}$ for 3 days) and again achieved CR. Donor lymphocyte infusion (DLI) was administered $\left(5 \times 10^{5} \mathrm{CD} 3+\right.$ cells/ $\mathrm{kg}$ in December 2014 and $1 \times 10^{6} \mathrm{CD} 3+$ cells/kg in April 2015). Despite this, the disease relapsed (molecular relapse) in August 2015. The patient received one cycle of low dose cytarabine while waiting for a suitable unrelated donor. Through September and October 2015 sequential stem cell transplantation was performed (HIDAC chemotherapy and then conditioning with treosulfan $12 \mathrm{mg} / \mathrm{kg}$ for 3 days, fludarabine $30 \mathrm{mg} / \mathrm{m}^{2}$ for 6 days and r-ATG [Thymoglobulin] $3 \mathrm{mg} / \mathrm{kg}$ for 2 days) with 9/10 human leukocyte antigen (HLA) mismatched unrelated male donor (DQB1 mismatch). 
Figure 1: FACS showing CD138 positivity, lambda restriction and CD20 negativity
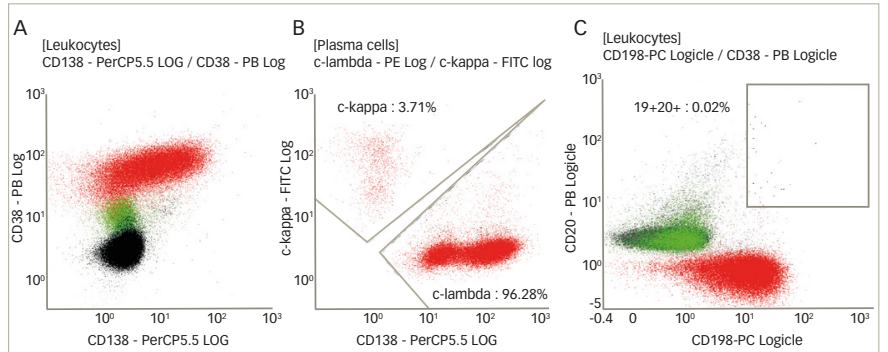

FACS $=$ fluorescence-activated cell sorting.

She was admitted on 14 December 2015 (day 66 after her second HSCT) for high fever, nausea and vomiting. The patient was given empirical treatment with meropenem. She showed no significant findings upon physical examination, on chest computed tomography (CT) scan, nor on abdominal ultrasound. Including normal liver, spleen and lymph nodes. No source of sepsis was found. Bone marrow aspirate confirmed remission of AML. Her EBV blood level was rising $\left(1.1 \times 10^{6}\right.$ copies $\left./ \mathrm{ml}\right)$. Peripheral blood fluorescence-activated cell sorting (FACS) showed $10 \%$ of monoclonal plasma cells (Figure 1). EBV PTLD was then diagnosed. The patient received rituximab $\left(375 \mathrm{mg} / \mathrm{m}^{2}\right.$ on days $1,8,15,22)$ as the first line of treatment on days 67 and 74 . Her EBV blood level decreased, but only temporarily (Figure 2). On day 74 the patient's condition worsened and she presented with melena. Gastroduodenoscopy with gastric and duodenal biopsy was performed. Duodenal biopsy showed EBV-positive PTLD (plasma cells, lambda light chain restricted, CD138 positive, most of them EBV positive using Epstein-Barr encoding region [EBER] in situ hybridisation [Figures 3 and 4]). The patient also presented with paraproteinaemia $(6.6 \mathrm{~g} / \mathrm{l})$ and elevated lambda free light chains $(1,038 \mathrm{mg} / \mathrm{l})$ on day 70 . Five days later she developed severe sepsis with renal impairment. Antibiotic therapy was escalated (linezolid) and renal replacement therapy was started. On day 87 the PTLD rapidly progressed: $29 \%$ monoclonal plasma cells in her blood via FACS, $4.35 \times 10^{6}$ EBV copies $/ \mathrm{ml}$ and further elevation of paraproteinaemia (9.2 g/l) was present. The failing rituximab monotherapy was replaced by the second-line treatment (rituximab $375 \mathrm{mg} / \mathrm{m}^{2}$ for 1 day, cyclophosphamide $600 \mathrm{mg} / \mathrm{m}^{2}$ for 1 day and methylprednisolone $2 \mathrm{mg} / \mathrm{kg}$ for 5 days). ${ }^{11}$ Her condition temporarily improved with disappearance of sepsis signs so antibiotic therapy was deescalated. On day 92 the patient was again septic with severe diarrhoea. Broad spectrum antibiotics were again administered (meropenem, linezolid, amikacin). The EBV blood levels were again rising rapidly (Figure 1). CHOP chemotherapy (cyclophosphamide, doxorubicin, vincristine and prednisone) was given as the third line of therapy on day 101. Despite all the treatment, the patient went into septic shock and died on 19 January, day 102.

\section{Discussion}

PTLD is a heterogeneous group of diseases and there are no strict guidelines for therapeutic approach. One of the options is reduction of immune suppression (RI) but it only occasionally results in $\mathrm{CR}$, and is mostly effective for early lesions. ${ }^{7}$ Rituximab can be used as the first line of therapy for most of the newly diagnosed polymorphic and monomorphic PTLDS and plays a prominent role in treatment. Several studies strongly suggest that early introduction of rituximab-based therapy significantly improves overall survival. ${ }^{9}$ Evens et al. performed a multicentre analysis of 80 patients with PTLD after solid organ transplant. A 3-year progressionfree survival and overall survival for patients who received firstline
Figure 2: Graphs showing trends of EBV, LDH, bilirubin and plasmatic cell levels in peripheral blood

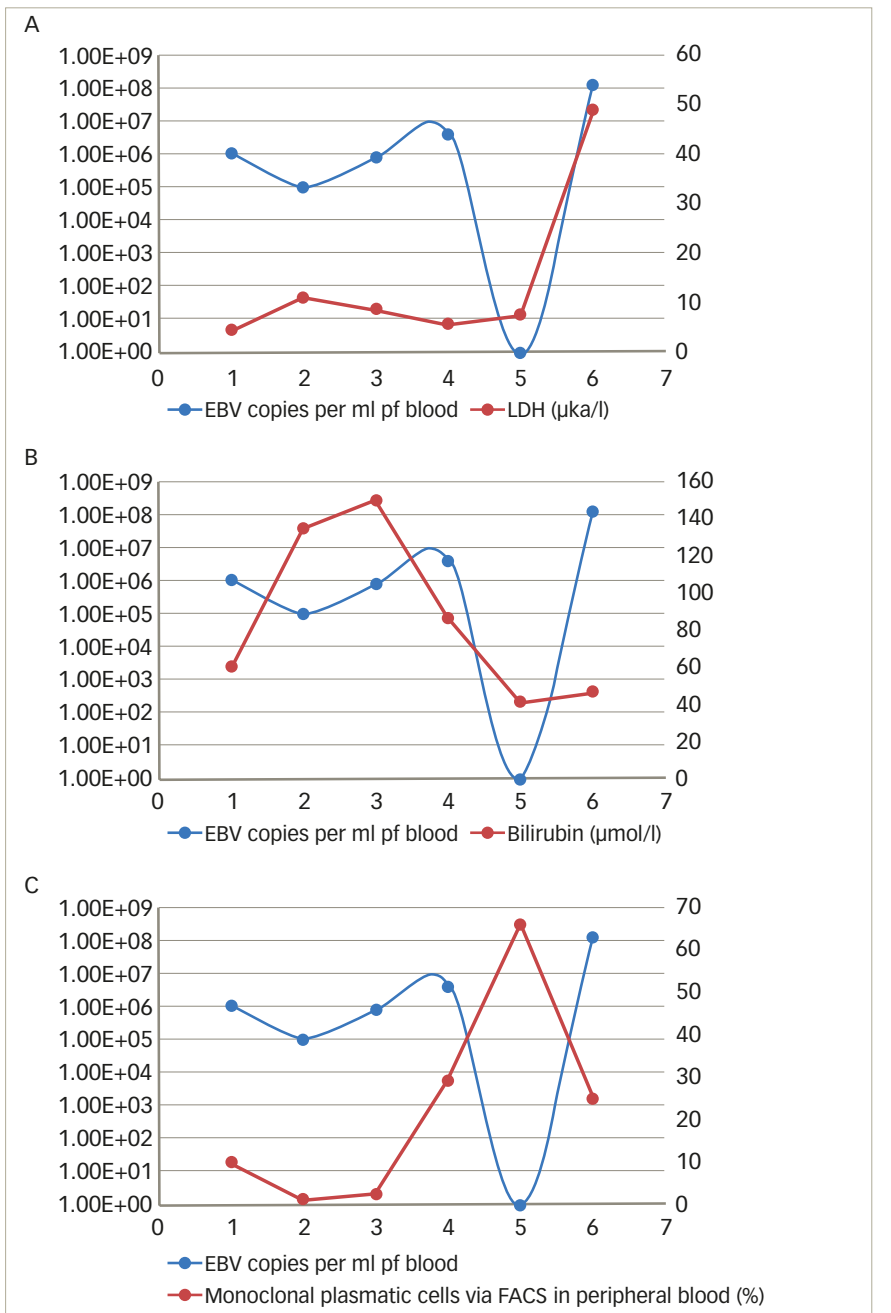

The trend of EBV copies in peripheral blood with $L D H(A)$, bilirubin (B) and monoclonal plasmatic cells (C). EBV = Epstein-Barr viruS; FACS = fluorescence-activated cell sorting; $L D H=$ lactate dehydrogenase.

Figure 3: Haematoxylin-eosin staining

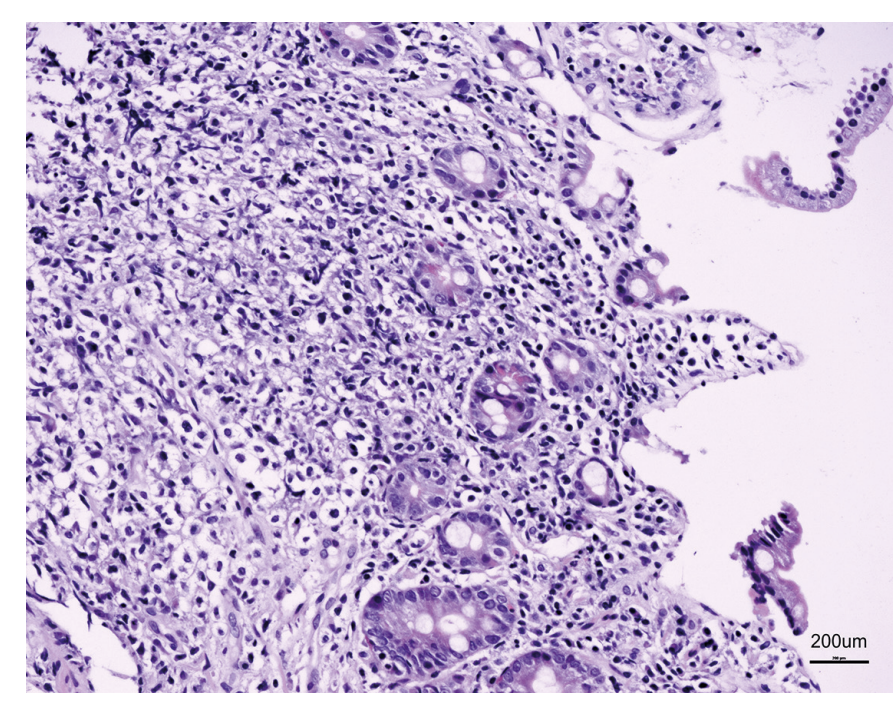

Image 100x - small tissue fragments of enteral mucosa with almost diffuse infiltration with mixture of medium-sized and larger cells.

rituximab-based therapy were $70 \%$ and $73 \%$, compared with $21 \%$ and $33 \%$ among patients who did not receive it as a component of initial therapy. ${ }^{12}$ 
Figure 4: CD138 immunohistochemical staining

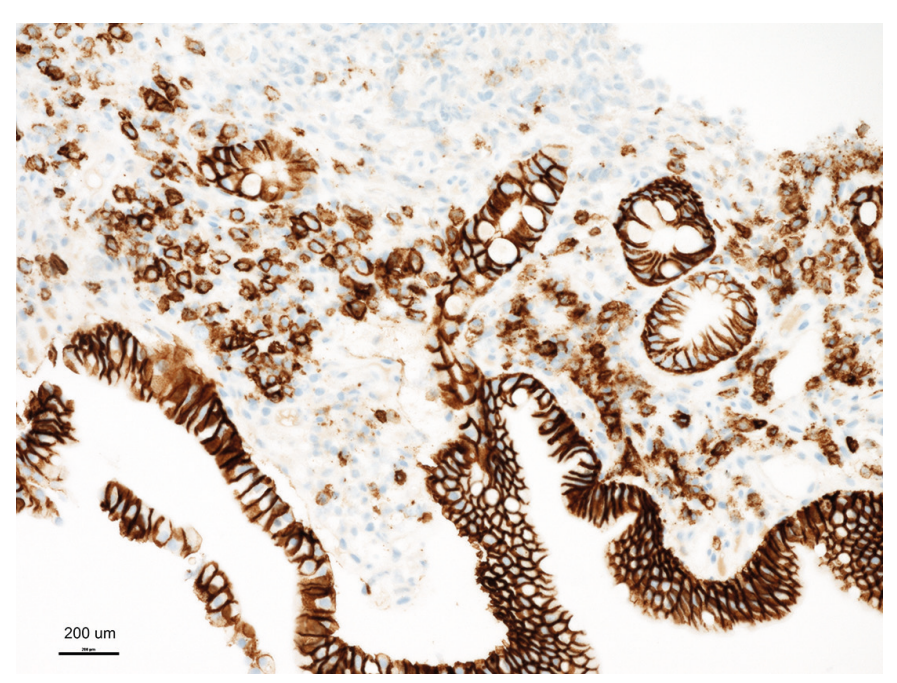

Image 100x - partial plasmacytoid differentiation is seen (positivity of epithelium is seen as well).

Also, rituximab is relatively safe with few side effects (especially compared to chemotherapy). ${ }^{6}$ Other options involve CHOP-like chemotherapy.

Our patient had PTLD with plasma cell differentiation, which is a rare type of PTLD. Karuturi et al. performed a retrospective analysis of 210 adult, solid-organ transplant, PTLD patients and only nine (4\%) had PTLD with plasma cell differentiation. All their patients presented with extranodal involvement and laboratory tests included elevated lactate dehydrogenase $(\mathrm{LDH})$, beta-2-microglobulin, present paraprotein and EBV-positive tumour. CD138 positivity and a CD20 negativity is characteristic for PTLD with plasma cell differentiation. Radiotherapy, resection, and RI appeared to have at least some effect in these patients. ${ }^{10}$ Trappe et al. performed a prospective study from 2006 to 2010 of patients diagnosed with plasmacytoma-like monomorphic PTLD after solid organ transplantation. One hundred and eighty-two patients were reported to the German registry in those years and only eight (4\%) had a diagnosis of monomorphic plasmacytoma-like PTLD. The cell population showed either lambda (two of eight patients) or kappa (six of eight patients) light chain restriction, and CD138 positivity (eight of eight), while CD20 was negative in all, and CD56 in seven of eight cases. Out of the six patients who received a RI as a first line of therapy two responded, and four patients showed progressive disease. The three patients with disseminated and progressive disease after RI, received systemic chemotherapy analogous to therapy of multiple myeloma. All three responded to treatment including one $\mathrm{CR}$ after chemotherapy consisting of bortezomib, doxorubicin and dexamethasone. One patient eventually died from disease progression. None of the patients received rituximab, as staining for CD20 was negative in all cases. ${ }^{7}$

Our patient's plasmatic cells were CD138 positive, CD20 negative, CD56 negative and showed lambda light chain restriction (Figure 2). The patient presented with LDH elevation and elevated EBV levels in blood. Elevated bilirubin levels correlated with activity of PTLD (Figure 1). Despite being CD20 negative the rituximab therapy was capable of reducing an amount of EBV blood levels and number of monoclonal plasma cells in blood, if only temporarily (Figure 1). As a second line of therapy the patient was given combination of rituximab, cyclophosphamide and methylprednisolone, a regimen used by Gross for patients who failed in first-line therapy for PTLD after solid-organ transplant. Overall, a response rate of $83 \%$ and CR of $75 \%$ was achieved in 36 patients. ${ }^{11}$ Our patient's EBV copies in blood temporarily lowered. But even this regimen helped only for a short period of time.

This example and another studies show beyond doubt that rituximab improved clinical outcome of many patients with PTLD, but it still remains ineffective for many patients. And plasmacytoma-like PTLD can be one of the examples. Other possibilities for treatment like EBV-specific cytotoxic represent promising strategies in improvement of survival of patients with PTLD. ${ }^{5}$ Whether we should use treatment similar to treatment for multiple myeloma (like proteasome inhibitors) to patients with plasmacytoma-like PTLD remains unclear and should be investigated. $\square$
Gottschalk S, Rooney CM, Heslop HE, Post-transplant lymphoproliferative disorders, Annu Rev Med, 2005;56:29-44.

2. Petrara MR, Giunco S, Serraino D, et al., Post-transplant Iymphoproliferative disorders: From epidemiology to pathogenesis-driven treatment, Cancer Letters, 2015;369:37-44.

3. Babcock GJ, Decker LL, Freeman RB, Thorley-Lawson DA, Epstein-Barr virus-infected resting memory B cells, not proliferating lymphoblasts, accumulate in the peripheral blood of immunosuppressed patients, J Exp Med, 1999;190:567-76.

Heslop HE, How I treat EBV Iymphoproliferation, Blood, 2009;114:4002-8.

5. Rouce RH, Louis CU, Heslop HE, EBV lymphoproliferative disease after hematopoietic stem cell transplant, Curr Opin Hematol, 2014;21:476-81.
6. Elstrom Rl, Andreadis $\mathrm{C}$, Aqui NA, et al., Treatment of PTLD with Rituximab or Chemotherapy, Am I Transplant. 2006;6:569-76

7. Trappe R, Zimmermann H, Fink S, et al., Plasmacytoma-like post-transplant lymphoproliferative disorder, a rare subtype of monomorphic B-cell post-transplant lymphoproliferation, is associated with a favorable outcome in localized as well as in advanced disease: a prospective analysis of 8 cases, Haematologica, 2011:96:1067-71.

8. Ibrahim HAH, Naresh KN, Ibrahim HAH, Naresh KN, Posttransplant lymphoproliferative disorders, Adv Hematol 2012;2012:e230173.

. Jagadeesh D, Woda BA, Draper J, Evens AM, Post transplant lymphoproliferative disorders: risk, classification, and therapeutic recommendations, Curr Treat Options in Oncol, 2012;13:122-36

10. 10. Karuturi M, Shah N, Frank D, et al., Plasmacytic posttransplant Iymphoproliferative disorder: a case series of nine patients, Transpl Int, 2013;26:616-22.

11. Gross TG, Bucuvalas JC, Park JR, et al., Low-dose chemotherapy for Epstein-Barr virus-positive post-transplantation lymphoproliferative disease in children after solid organ transplantation, J Clin Oncol, 2005;23:6481-8.

12. Evens AM, David KA, Helenowski I, et al., Multicenter analysis of 80 solid organ transplantation recipients with post-transplantation lymphoproliferative disease: outcomes and prognostic factors in the modern era, J Clin Oncol, 2010;28:1038-46. 